\title{
The experience of endotracheal intubation in Middle East respiratory syndrome patients -A case report-
}

\author{
Department of Anesthesiology and Pain Medicine, National Medical Center, Seoul, Korea
}

Mi-Young Kwon, Gunn Hee Kim, Byunguk Kim, and Min Seok Koo

After Middle East respiratory syndrome (MERS) was first confirmed in Korea on May 20, 2015, a total of 186 confirmed cases and 37 deaths occurred until the announcement of its cessation on December 23, 2015. MERS often causes severe pneumonia; accordingly, many patients require endotracheal intubation for mechanical ventilation. At our hospital, we treated 30 confirmed and 29 suspected cases and performed 9 endotracheal intubations in 8 of these patients, using conventional direct laryngoscopy (DL) and GlideScope video-laryngoscopy (GL). We faced difficulty in conducting endotracheal intubation due to the personal protective equipment and the limited bed height required for managing MERS patients. In such cases, GL improved the ease and direct confirmation of success of endotracheal intubation as compared to DL. In addition, we found that portable end-tidal $\mathrm{CO}_{2}$-monitoring devices may facilitate more precise and reliable confirmation of successful intubation. (Anesth Pain Med 2017; 12: 191-194)

Key Words: Airway management, Intubation, Middle East respiratory syndrome coronavirus, Personal protective equipment.

After the introduction of Middle East respiratory syndrome (MERS) into Korea had been confirmed on May 20, 2015, a total of 186 confirmed cases and 37 deaths occurred in the period up to the announcement of cessation on December 23,

Received: July 26, 2016.

Revised: November 9, 2016.

Accepted: November 21, 2016

Corresponding author: Gunn Hee Kim, M.D., Ph.D., Department of Anesthesiology and Pain Medicine, National Medical Center, 245, Eulji-ro, Jung-gu, Seoul 04564, Korea. Tel: 82-2-2260-7372, Fax: 82-2-2262-4766, E-mail: gunnhee@gmail.com

It was presented The 92th Annual Scientific Meeting of the Korean Society of Anesthesiologists, November 2015, Bexco, Busan, Korea.

This is an Open Access article distributed under the terms of the Creative Commons Attribution Non-Commercial License (http://creativecommons.org/licenses/by-nc/4.0) which permits unrestricted non-commercial use, distribution, and reproduction in any medium, provided the original work is properly cited.
2015 [1]. Various symptoms ranging from asymptomatic infection to serious symptoms of septic shock and multi-organ failure can appear in patients infected with the MERS coronavirus (MERS-coV). In particular, the MERS-coV causes severe pneumonia in many cases by invading the lungs; these cases often require endotracheal intubation for mechanical ventilation [2,3]. Healthcare professionals are reportedly most frequently exposed to MERS-coV while conducting intubation in the infected patients [4]; thus, these professionals wear personal protective equipment that can provide the highest level of protection and then perform endotracheal intubation with limited staff in negative-pressure isolation rooms. Accordingly, endotracheal intubation in these patients requires special care and consideration as compared to airway management conducted in intensive care units or operating rooms. While treating MERS patients in Korea, we performed intubation in 8 patients and here report on this experience, along with providing a review of the literature.

\section{CASE REPORT}

At our hospital, we treated a total of 59 MERS patients, including 30 confirmed and 29 suspected cases. The MERS-CoV caused severe pneumonia in many cases and 8 patients progressed to acute respiratory distress syndrome. These cases required endotracheal intubation for mechanical ventilation. Since 1 patient required 2 intubations, spaced over time, we performed a total of 9 endotracheal intubations.

When endotracheal intubation was found to be necessary while the MERS patients received treatment under isolation in negative-pressure rooms, the medical attendant called on the procedure team, which included a surgical and an anesthesia specialist. Our hospital procedure teams are responsible for 
various procedures, including endotracheal intubation, central venous cannulation, and arterial cannulation, while being on 24-h rotational shifts.

Our healthcare professionals wore Level $\mathrm{C}$ personal protective equipment (PPE) when conducting procedures, during which aerosols can form, on confirmed MERS patients (Fig. 1). They wore a detachable powered air-purifying respirator (PAPR [S-533L, 3M, USA]), a hooded chemical splash-resistant coverall with integrated foot cover (Microchem 3000, 3M, USA), inner and outer chemical- resistant gloves, and inner and outer chemical-resistant shoe covers.

Nine endotracheal intubations were performed by 5 specialists experienced in anesthesia, internal medicine, neurosurgery, emergency medicine, and thoracic surgery. After forming groups of 2 and wearing PPE in advance, the team carried the drugs and equipment required for endotracheal intubation into the negative-pressure room, where the patient was attended. One person set the mechanical ventilator in the room to suit the patient's conditions in advance and the other person, to make the procedure more convenient, placed the required drugs and equipment, in a particular order, next to the patient. All patients received standard monitoring, including electrocardiogram, noninvasive blood pressure, pulse oximetry monitoring. The team first administered sufficient $100 \%$ oxygen for preoxygenation and midazolam $0.1 \mathrm{mg} / \mathrm{kg}$ or propofol 0.2 $\mathrm{mg} / \mathrm{kg}$ was administered for sedation. Loss of consciousness was verified by the inability to communicate; then, the team sequentially administered neuromuscular blocking agents
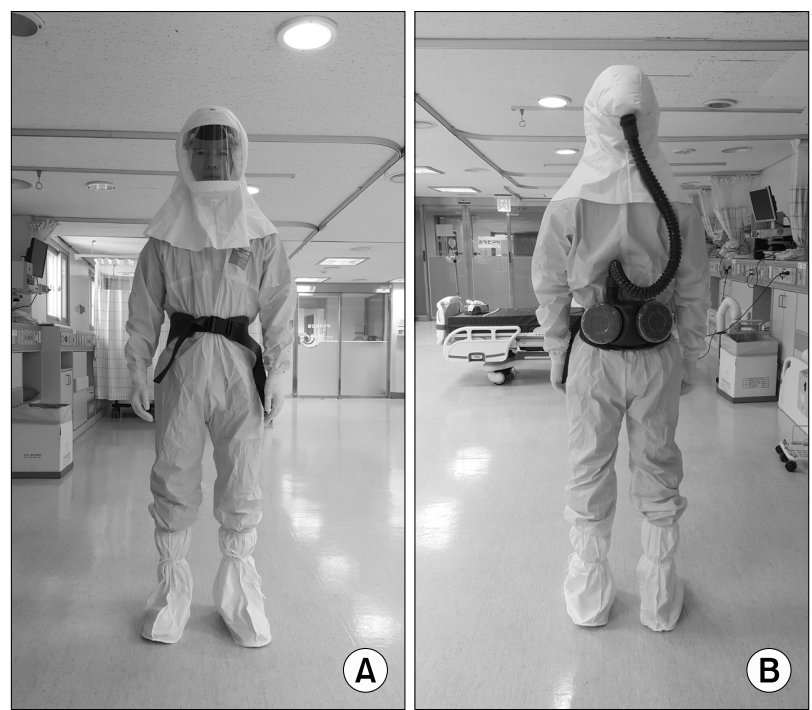

Fig. 1. Level $\mathrm{C}$ personal protective equipment in frontal view (A) and back view (B). (succinylcholine $1.0 \mathrm{mg} / \mathrm{kg}$ or vecuronium $0.1 \mathrm{mg} / \mathrm{kg}$ ) and opioids, such as fentanyl, depending on the patient's response. Depending on the preference of the specialist performing the endotracheal intubation, either conventional direct laryngoscopy (DL) or GlideScope video-laryngoscopy (GL, Verathon Inc., USA) was used, and the team performed endotracheal intubation using GL if they determined during the procedure that the intubation was problematic. The endotracheal intubation method used, whether it was successful, the number of trials, and the Cormak-Lehane grade of each patient are shown in Table 1 .

Among a total of 9 endotracheal intubations, 5 endotracheal intubations conducted using GL were successful. Among these, 2 were conducted using GL from the start and were successful in a single trial. For the other 3 cases, the initial DL intubation failed; therefore, the procedure team switched to GL, after which intubation succeeded in a single trial.

Since the procedure team could not use stethoscopes while wearing PPE, determination of the success of endotracheal intubation was achieved by directly confirming that the tube passed through the vocal cords and confirming the formation of condensation on the tube during exhalation. A final confirmation of successful intubation was conducted by verifying on chest X-ray that the tube was located in the trachea.

\section{DISCUSSION}

MERS was first reported in June 2012 when a patient in Saudi Arabia died from a severe respiratory disease; the condition is characterized by symptoms of asymptomatic

Table 1. Patients' Intubation Methods and Trial Numbers

\begin{tabular}{|c|c|c|c|c|c|}
\hline \multirow{2}{*}{ No. } & \multirow{2}{*}{ Age } & \multirow{2}{*}{ Sex } & \multicolumn{2}{|c|}{ Intubation trial number } & \multirow{2}{*}{$\begin{array}{c}\text { Cormak-Lehan } \\
\text { grade }\end{array}$} \\
\hline & & & Conventional & GlideScope & \\
\hline 1 & 71 & M & 2 & 1 & $3-4$ \\
\hline 2 & 56 & M & 0 & 1 & 4 \\
\hline 3 & 86 & M & 3 & 0 & $3-4$ \\
\hline 4 & 68 & $M$ & 1 & 1 & 3 \\
\hline 5 & 68 & M & 0 & 1 & 3 \\
\hline 6 & 52 & $\mathrm{~F}$ & 1 & 1 & 3 \\
\hline 7 & 71 & $M$ & 1 & 0 & 3 \\
\hline 8 & 45 & M & 1 & 0 & 2 \\
\hline 9 & 86 & $M$ & 1 & 0 & 2 \\
\hline
\end{tabular}

Number 4 and 5 are same patient. The patient required 2 intubations at a distance of time. 
infection, severe pneumonia with acute respiratory distress syndrome, septic shock, and multi-organ failure, which results in death [2]. Since MERS-coV often causes severe pneumonia, endotracheal intubation is often required for mechanical ventilation [3]. Additionally, at our hospital, endotracheal intubation was required in 8 of 59 confirmed and suspected cases; this shows that endotracheal intubation is often necessary for the treatment of MERS.

Among endotracheal intubations that are regularly performed in operating rooms by doctors with sufficient experience in airway management, difficult endotracheal intubation has a frequency as low as $0.7-2.3 \%$ [5]. In endotracheal intubation conducted in operating rooms, the patient's airway condition most often contributes to the difficulty of intubation. However, when performing endotracheal intubation in MERS patients in negative-pressure rooms, environmental factors, such as the PPE of the doctor and the fixed height of the bed, also contribute to the difficulty, in addition to the patients' airway conditions. Beds were placed at a fixed height in a negativepressure isolation room at the outset, and could not be changed, despite necessity, due to the possibility of viral transmission. Because the height of the bed could not be adjusted, the doctor had to bend his or her body markedly. However, the PPE limits bending of the body, as the oxygen line connecting into the PAPR at the waist can be pulled in the process. Moreover, excessive bending can also cause disconnection of the oxygen line (Fig. 2A). In addition, because the doctor wore facial protective equipment, he/she had a narrower view during the procedure; similarly, because the moisture in the doctors' exhaled air condensed on the facial protective equipment, the doctor's view was further limited.

While reviewing the cases treated at our hospital, we learned that there is an advantage to using the GL for endotracheal intubation when the doctor wears Level C PPE. When using DL, the doctor had to bend at the waist to view the glottis in a straight line while holding the laryngoscope. In contrast, the GL enabled the doctor to gain a better glottic view while in an upright, standing position and thus the disconnection of the PAPR oxygen line could be prevented. Moreover, compared to when using DL, the risk of droplet infection is likely to decrease when the GL is used, because it allows for a relatively greater distance between the doctor and the patient's face (Fig. 2B).

GL was also useful for confirming whether the endotracheal intubation was successful. To determine whether endotracheal intubation is successful, it is most important to confirm the exact location of the tube after intubation. The most precise method for confirmation of successful endotracheal intubation is direct visual confirmation of whether the tube is inserted through the glottis. However, in difficult endotracheal intubation, direct confirmation by eye is hampered. In operating rooms, esophageal intubation can be detected early through monitoring of end-tidal $\mathrm{CO}_{2}$ immediately after intubation, thus decreasing the occurrence of complications $[6,7]$. However, unlike in operating rooms, end-tidal $\mathrm{CO}_{2}$ could not be monitored in negative-pressure isolation rooms, and we therefore had to use a different indirect method. In general, methods for indirectly confirming the location of the endotracheal tube include checking the respiratory sound by auscultating the chest and stomach, confirming chest movement, abdominal distension, and condensation of water on the inner surface of the tube, confirming whether oxygen saturation of the patient is well maintained, and confirming the elasticity of the endotracheal tube cuff [8]. However, because the doctors could not auscultate MERS patients while wearing PPE, successful intubation was verified by performing endotracheal intubation using the GL, which allowed the doctors to have a clear glottic view.

Based on this MERS outbreak crisis, we have identified several issues that healthcare professionals should keep in mind. Airway management is essential in treating critically ill MERS patients. Complications occur at a high frequency
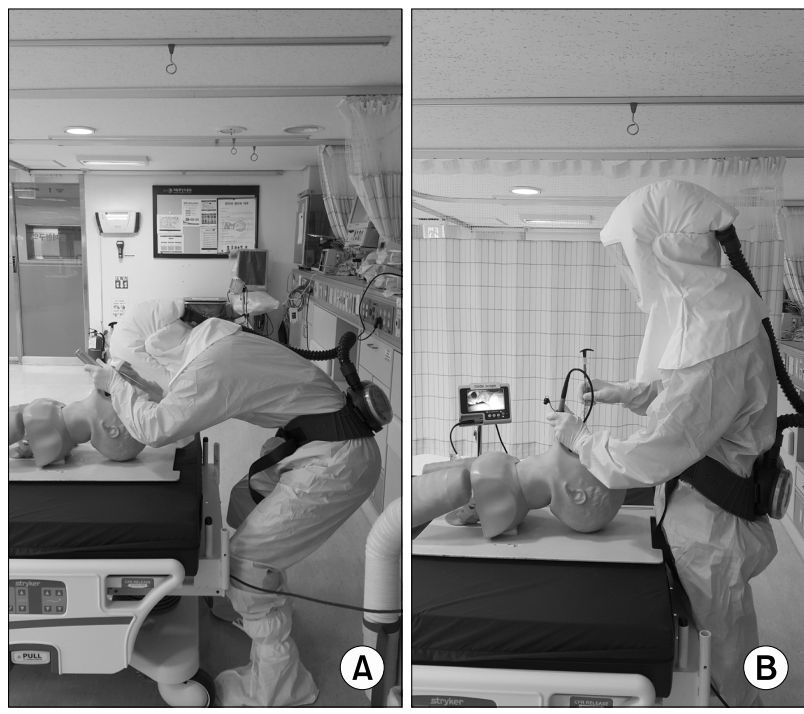

Fig. 2. Comparison of conventional direct laryngoscopy (A) and GlideScope video-laryngoscopy (B). 
during emergency endotracheal intubation that is performed in patients with respiratory distress and shock, or in critically ill patients who require cardiopulmonary resuscitation [9]. Since a greater burden is placed on the doctor when the procedure is conducted while wearing PPE, the patient's situation should be identified in advance, and the medical attendant should share opinions with the procedure team to avoid emergency endotracheal intubation in MERS patients.

At our hospital, because the only healthcare professionals who could use the GL were anesthesia and emergency medicine specialists, the use of GL was limited. In future, healthcare professionals who treat critically ill patients should be well informed of various endotracheal intubation methods, besides DL.

End-tidal $\mathrm{CO}_{2}$ measurement is perhaps one of the most reliable means of determining proper endotracheal tube placement [10]. At our hospital, end-tidal $\mathrm{CO}_{2}$ could not be monitored in negative-pressure isolation rooms due to the absence of the required devices. We recommend that end-tidal $\mathrm{CO}_{2}$ monitoring devices should be included in isolation room equipment for confirmation of successful intubation.

Many previous studies have compared the use of GL and DL for endotracheal intubation. However, studies have rarely compared these approaches in situations that require PPE. Aberle et al. [11] have compared DL and GL while wearing PPE, using mannequins as subjects, but concluded that there was no difference in the time required for endotracheal intubation or in the ease of use. Moreover, Shin et al. [12] compared DL and the Pentax-Airwayscope (AWS, Pentax Corporation, Japan), which is a type of portable video laryngoscope, while wearing or not wearing PPE, and using mannequins as subjects. Significant improvements in terms of the time required for intubation and the ease of endotracheal intubation were observed in the experimental group in which the Pentax-AWS was used, while wearing PPE, as compared to the experimental groups in which DL was used, while wearing or not wearing PPE. Since the aforementioned studies were all conducted using mannequins as subjects, and not on actual patients, it remained unknown whether the same results would be observed in the clinic. In future, the GL should be compared with other endotracheal intubation methods under these circumstances.

In our hospital, portable chest X-ray is usually used to confirm whether the endotracheal tube is located in the right position. However, under special circumstances, such as when the patient is quarantined and needs to be intubated in an isolated area, it may take longer to assess the position of the endotracheal tube. GL may be helpful in such cases, but requires portable end-tidal $\mathrm{CO}_{2}$ monitoring for more precise and reliable confirmation of successful intubation. Therefore, it is suggested that portable end-tidal $\mathrm{CO}_{2}$ monitoring be used when intubating patients who have an infection that requires the physician to wear PPE.

\section{REFERENCES}

1. KCDC. Middle East Respiratory Syndrome Coronavirus Outbreak in the Republic of Korea, 2015. Osong Public Health Res Perspect 2015; 6: 269-78.

2. Zumla A, Hui DS, Perlman S. Middle East respiratory syndrome. Lancet 2015; 386: 995-1007.

3. Arabi YM, Arifi AA, Balkhy HH, Najm H, Aldawood AS, Ghabashi A, et al. Clinical course and outcomes of critically ill patients with Middle East respiratory syndrome coronavirus infection. Ann Intern Med 2014; 160: 389-97.

4. Baba H, linuma $Y$, Imaizumi $K$, Hasegawa $Y$, Hasegawa $T$, Ohta $\mathrm{M}$, et al. Transmission of bacterial infections to healthcare workers during intubation and respiratory care of patients with severe pneumonia. Infect Control Hosp Epidemiol 2009; 30: 1019-21.

5. Wilson ME, Spiegelhalter D, Robertson JA, Lesser P. Predicting difficult intubation. Br J Anaesth 1988; 61: 211-6.

6. Mort TC. Esophageal intubation with indirect clinical tests during emergency tracheal intubation: a report on patient morbidity. J Clin Anesth 2005; 17: 255-62.

7. Ku PK, Tong MC, Ho KM, Kwan A, van Hasselt CA. Traumatic esophageal perforation resulting from endotracheal intubation. Anesth Analg 1998; 87: 730-1.

8. O'Connor RE, Swor RA. Verification of endotracheal tube placement following intubation. National Association of EMS Physicians Standards and Clinical Practice Committee. Prehosp Emerg Care 1999; 3: 248-50.

9. Schwartz DE, Matthay MA, Cohen NH. Death and other complications of emergency airway management in critically ill adults. A prospective investigation of 297 tracheal intubations. Anesthesiology 1995; 82: 367-76.

10. Varon AJ, Morrina J, Civetta JM. Clinical utility of a colorimetric end-tidal $\mathrm{CO} 2$ detector in cardiopulmonary resuscitation and emergency intubation. J Clin Monit 1991; 7: 289-93.

11. Aberle SJ, Sandefur BJ, Sunga KL, Campbell RL, Lohse CM, Alecastro Puls H, et al. Intubation Efficiency and Perceived Ease of Use of Video Laryngoscopy vs Direct Laryngoscopy While Wearing HazMat PPE: A Preliminary High-fidelity Mannequin Study. Prehosp Disaster Med 2015; 30: 259-63.

12. Shin DH, Choi PC, Na JU, Cho JH, Han SK. Utility of the Pentax-AWS in performing tracheal intubation while wearing chemical, biological, radiation and nuclear personal protective equipment: a randomised crossover trial using a manikin. Emerg Med J 2013; 30: 527-31. 